\title{
Scalable population estimates using spatial-stream-network (SSN) models, fish density surveys, and national geospatial database frameworks for streams
}

\begin{tabular}{|r|l|}
\hline Journal: & Canadian Journal of Fisheries and Aquatic Sciences \\
\hline Manuscript ID & cjfas-2016-0247.R2 \\
\hline Manuscript Type: & Rapid Communication \\
\hline Date Submitted by the Author: & 05-Nov-2016 \\
\hline Keywote List of Authors: & $\begin{array}{l}\text { Isaak, Daniel; USDA Forest Service } \\
\text { Ver Hoef, Jay; National Marine Fisheries Service - NOAA, Alaska Fisheries } \\
\text { Science Center } \\
\text { Peterson, Erin; Queensland University of Technology } \\
\text { Horan, Dona; USDA Forest Service Rocky Mountain Region } \\
\text { Nagel, David; USDA Forest Service }\end{array}$ \\
\hline \multirow{2}{*}{ spatial-stream network, block-kriging, fish density, population estimate, } \\
geostatistics
\end{tabular}

\section{SCHOLARONE \\ Manuscripts}


2 Scalable population estimates using spatial-stream-network (SSN) models, 3 fish density surveys, and national geospatial database frameworks for streams 4 5

6 Daniel J. Isaak $^{1 *}$, Jay M. Ver Hoef ${ }^{2}$, Erin E. Peterson ${ }^{3}$, Dona L. Horan ${ }^{1}$, David E. Nagel ${ }^{1}$ 7 8

$9 \quad{ }^{1}$ U.S. Forest Service Rocky Mountain Research Station, Boise ID 83702

10

$11{ }^{2}$ Marine Mammal Laboratory, NOAA-NMFS Alaska Fisheries Science Center, 7600 Sand Point Way NE, 12 Seattle, WA 98115

13

${ }^{3}$ ARC Centre of Excellence for Mathematical and Statistical Frontiers and the Institute for Future Environments, Queensland University of Technology (QUT), QLD, Australia

16 
19 Abstract: Population size estimates for stream fishes are important for conservation and 20 management but sampling costs limit the extent of most estimates to small portions of river 21 networks that encompass 100s-10,000s of linear kilometers. However, the advent of large fish

22 density datasets, spatial-stream-network (SSN) models that benefit from non-independence

23 among samples, and national geospatial database frameworks for streams provide the

24 components to create a broadly scalable approach to population estimation. We demonstrate such

25 an approach with trout density surveys from 108 sites in a 735 kilometer river network.

26 Universal kriging was used to predict a continuous map of densities among survey locations and 27 block kriging (BK) was used to summarize discrete map areas and make population estimates at 28 stream, river, and network scales. The SSN models also accommodate covariates, which 29 facilitates hypothesis testing and provides insights about factors affecting patterns of abundance.

30 The SSN-BK population estimator can be applied using free software and geospatial resources to 31 develop valuable information at low cost from many existing fisheries datasets.

32

33 Keywords: spatial-stream-network, block kriging, fish density, population estimate, geospatial 


\section{Introduction}

Answering the question "How many fish live in that stream, river, or lake?" is of fundamental importance to fisheries management and species conservation efforts. Estimation methods addressing that question form an extensive literature and many sampling techniques have been developed to collect datasets for use with estimators (Hilborn and Walters 1992; Zale et al. 2013). In lotic systems, fish are often sampled by electrofishing, angling, or snorkeling (Dunham et al. 2009) and population estimates are obtained for short reaches of stream using mark-recapture (Peterson 1896; Lincoln 1930) or depletion-removal estimators (Zippin 1958). For nest-building species like salmon and trout, it is also common to conduct visual surveys during the spawning season and use nest counts as a density index or measure of population size (Al-Chokhachy et al. 2005; Falke et al. 2013). Collectively, those estimates form the core datasets that state and federal management agencies use to monitor the status and trends of many species and fisheries throughout North America and Europe. Thousands of stream and river sites have been sampled in previous decades to estimate local population sizes (Wenger et al. 2011; Meyer et al. 2013; Millar et al. 2016) and as these databases grow, so too do opportunities to mine them for novel information (Isaak et al. 2014).

What is considered a "population" when applying traditional estimators to short sections of stream rarely matches the spatial scales at which habitats are occupied by reproducing populations. Most reproducing populations of stream fishes occupy 1s-10s of network kilometers and are affected by natural gradients and anthropogenic stressors occurring over similar scales (Schlosser 1991). The mismatch between measurement scale and biological reality lies at the heart of the Riverscapes paradigm articulated by Fausch et al. (2002) and creates the fundamental need for spatially continuous broad-scale information to better understand and conserve freshwater fishes. Spatial sampling strategies like that espoused by Hankin and Reeves (1988) or more recent attempts (Stevens and Olsen 2004; Torgerson et al. 2006; Korman et al. 2016) partially address information needs but are costly and difficult to implement in hundreds of streams throughout the ranges of species or across the 100s-10,000s of linear kilometers that constitute river networks. Another critical and largely unrecognized impediment to developing spatial fisheries information has been the lack of consistent geospatial database frameworks for streams to enable efficient organization, summarization, and sharing of data within or among agencies (Cooter et al. 2010). Such frameworks would provide a database structure wherein each 
stream reach within a river network is assigned a unique identifier, attributed with topological information (e.g., up- and downstream flow-routing), and georeferenced in a cartographic projection system. Networks with those properties could bridge between relational databases (e.g., Access or Oracle) that are used to store large fisheries datasets and geographic information systems (GIS) that would be used to manipulate and visualize data associated with broadscale population estimation. Also required are flexible analytical tools for data collected from stream networks, especially those capable of accommodating the clustered, non-independent sample locations that inevitably arise during the history of resource agencies or when data are aggregated from multiple sources.

In recent years, key statistical and technical advances addressed many of the preceding issues to provide the basic elements for creating a broadly scalable approach to population estimation. The development of spatial-stream-network (SSN) models (Ver Hoef et al. 2006; Ver Hoef and Peterson 2010) based on covariance structures for network topology (Peterson and Ver Hoef 2010) and that rely on assumptions about the stochastic processes generating observable data (Schabenberger and Gotway 2005), facilitates valid inference from non-independent stream samples. As extensions of the linear-mixed model, SSNs accommodate covariates to describe relationships with response variables and simulation studies indicate their accuracy in fixed effect parameter estimation and confidence interval coverage for a wide range of conditions (Som et al. 2014; Rushworth et al. 2015). Concerns have been raised about "spatial confounding" in the estimation of fixed effect parameters (Hodges and Reich (2015) but see Hanks et al. (2015) for a counter-argument) but such confounding is of limited relevance for making accurate spatial predictions. Like other spatial statistical models (Ver Hoef 2002; Beale et al. 2010; Temesgen and Ver Hoef 2015), SSNs consistently improve predictive performance relative to non-spatial models when used with spatially dense datasets that contain non-independent samples (Isaak et al. 2010; Brennan et al. 2016; Turschwell et al. 2016). Classical geostatistical techniques (Cressie 1993) have also been adapted for implementation with the SSN models based on stream distances rather than Euclidean distances, which enables kriging and blockkriging predictions to be made throughout river networks with spatially explicit errors (Ver Hoef et al. 2006).

Paralleling the development of SSN models has been the development of nationally consistent geospatial frameworks for stream data (Cooter et al. 2010; Moore and Dewald 2016). 
Most notably for Canada, lotic systems are represented by the National Hydro Network (NHN; http://ftp2.cits.rncan.gc.ca/pub/geobase/official/nhn_rhn/doc/NHN.pdf), and within the United States by the National Hydrography Dataset (NHD; www.horizonsystems.com/NHDPlus/NHDPlusV2 home.php). The NHD is available in several resolutions, but of particular value is the medium resolution version (1:100,000-scale) because of the reach descriptor variables (e.g., elevation, slope, watershed size, and many others) that have been incorporated as Value Added Attributes to create NHDPlus (McKay et al. 2012). The reach descriptors can be used to query stream networks, visualize results within a GIS, and as covariates in predictive models. As the user-community associated with NHDPlus has grown, dozens of additional reach descriptors have been developed by groups like the National Fish Habitat Partnership (Wang et al. 2011) and the Environmental Protection Agency (Hill et al. 2016).

In this paper, we integrate SSN models and the geospatial resources described above with a fish density dataset to develop a scalable approach to population estimation. Models that predict fish density are developed based on different combinations of covariates and autocovariance functions that account for non-independence among samples. The models are used to predict continuous density maps, which are then summarized to make population estimates at stream, river, and network scales. For comparison to non-spatial analogues, estimates are also made using multiple linear regression (MLR) and simple random sampling (SRS). The dataset and statistical code used in the analysis are included as supplemental materials so that interested readers may explore these topics in detail.

\section{Materials and methods}

\section{Study area and dataset}

A dataset of trout density estimates at 108 sites was obtained from the $2,300 \mathrm{~km}^{2}$ Salt River watershed on the border between Idaho and Wyoming in the western U.S. The area is mountainous and 11 major tributaries drain north-south trending ranges at the eastern and western extents of the watershed (Figure 1). Tributaries and several spring streams that originate from the main valley floor were sampled at 104 locations during summer low-flow conditions (stream widths: 1.2-8.8 m, reach lengths: 63-465 m) in 1996 and 1997 by electrofishing within block-netted reaches to obtain local population estimates for age-1+ trout using depletion 
methods (Zippin 1958; Isaak and Hubert 2004). Samples were spaced at 50-m elevation intervals along most tributaries with additional samples taken near tributary confluences or upstream and downstream of abrupt contrasts in channel slope. Those data were supplemented with population estimates from four sites on the Salt River mainstem (river widths: 20 - $32 \mathrm{~m}$, reach lengths: 4.4-4.8 km) that were repeated in 1995, 1996, and 1998 by raft electrofishing using markrecapture methods (Pollock et al. 1990; Gelwicks et al. 2002). For current purposes, the Salt River estimates were averaged across years. Species composition, based on approximately 5,000 trout captured at the 108 sites, was $82.6 \%$ native Yellowstone cutthroat trout (Oncorhynchus clarkii bouvieri), 12.7\% non-native brown trout (Salmo trutta), 4.6\% non-native brook trout (Salvelinus fontinalis), and $0.1 \%$ non-native rainbow trout (O. mykiss). Population estimates at the 108 sites were standardized as trout $100 \mathrm{~m}^{-1}$ length of stream. Additional details about the dataset and study area are provided elsewhere (Gelwicks et al. 2002; Isaak and Hubert 2004).

A digital stream network for the NHD processing unit (Pacific Northwest 17) that encompassed the Salt River watershed was downloaded from the National Stream Internet website (NSI; www.fs.fed.us/rm/boise/AWAE/projects/NationalStreamInternet.html; Isaak et al. 2013) and clipped using the watershed boundary. The NSI network is derived from the 1:100,000-scale NHDPlus Version 2 network, has been topologically adjusted to facilitate SSN analysis using the Spatial Tools for the Analysis of River Systems software (STARS; Peterson and Ver Hoef 2014), and is available for all streams and rivers in the coterminous U.S. A one-toone relationship between reaches in the NSI and NHDPlus networks facilitates the use of NHD reach descriptors as covariates in SSN models. Here, we considered only a small number of covariates (reach slope, summer temperature, and stream canopy density), which have previously been associated with trout densities (Chisholm and Hubert 1986; Fausch et al. 1988; Isaak and Hubert 2004) and were available as reach descriptors in geospatial formats (Table 1). The NHD and NSI networks contain many reaches that do not support fish populations because of intermittent flow or excessive steepness, so the original Salt River network of 1,901 km was trimmed to a $735-\mathrm{km}$ network prior to analysis by deleting reaches with $>10 \%$ slope, those coded as intermittent in the NHDPlus dataset (e.g., Fcode = 46003), and based on observations made by the lead author during field sampling. We processed the final dataset using the current version of STARS (Peterson and Ver Hoef 2014, Version 2.0.4 downloaded from the SSN/STARS website: www.fs.fed.us/rm/boise/AWAE/projects/SpatialStreamNetworks.shtml) and output the spatial, 
158

159

160

161

162

163

164

165

166

167

168

$$
\gamma(h)=\frac{1}{2 N(h)} \sum_{\left\|s_{i-} s_{j}\right\| \in c(h)}\left[y\left(s_{i}\right)-y\left(s_{j}\right)\right]^{2},
$$
to conduct all subsequent analyses. locations, then an empirical estimator of the semivariance, $\gamma(h)$, is

topological, and attribute information as a Landscape Network object (LSN; available as Supplemental A) suitable for spatial analysis. The SSN package (Ver Hoef et al. 2014; Version 1.1.7) for the R statistical software (R Development Core Team 2014) was downloaded from the Comprehensive R Archive Network website (www.r-project.org/) and used with the LSN object

To describe spatial similarity, often referred to as autocorrelation, in the trout density dataset, a type of semivariogram called a Torgegram was initially calculated (Zimmerman and Ver Hoef 2017). The semivariance is one-half of the average squared difference between random variables separated by some intervening distance (Matheron 1963). If $s_{i}$ and $s_{j}$ contain the spatial coordinates for the $i$ th and $j$ th locations, and $y\left(s_{i}\right)$ and $y\left(s_{j}\right)$ are the measured values at those

where $h$ is the distance, $\left\|s_{i}-s_{j}\right\|$, between locations, $c(h)$ is the distance bin representing the interval around $h$ (chosen to be mutually exclusive and exhaustive so that all distances $h$ fall into one of the bins), and $N(h)$ is the number of data pairs $\left(s_{i}, s_{j}\right)$ in distance bin $c(h)$. The semivariogram provides a graphical representation of spatial autocorrelation in the measured data; when semivariance values are low (high) it indicates that sample pairs within a distance bin are similar (dissimilar). If positive autocorrelation occurs within a dataset, the semivariance values are smallest at short distance lags and increase as distance increases. The Torgegram is similar to a traditional semivariogram except that semivariance values are plotted separately for site-pairs with flow-connected (e.g. water flows from an upstream site through a downstream site) and flow-unconnected (e.g. sites reside on the same network but do not share the same flow) relationships because these patterns usually differ on stream networks (Peterson et al. 2013; Zimmerman and Ver Hoef 2017). As expected, given the density of the trout samples, the Torgegram showed strong similarities among site estimates in close proximity and weaker similarities as separation distances increased (Figure 2). Semivariance among flow-unconnected sites plateaued at approximately $10 \mathrm{~km}$ while semivariance among flow-connected sites steadily increased to the maximum distance of $50 \mathrm{~km}$. Those patterns indicated that trout densities became dissimilar among adjacent headwater streams (i.e., flow-unconnected relationships) over 
186

187

188

189

190

191

192

193

194

(2)

$$
\boldsymbol{y}=\mathbf{X} \beta+\mathbf{z}_{\mathrm{TU}}+\mathbf{z}_{\mathrm{TD}}+\mathbf{z}_{\mathrm{EUC}}+\boldsymbol{\varepsilon},
$$

195

196

197

198

199

200

201

202

203

204

205

206

207

208

209

210

211

212

213

214 to the river mainstem.

\section{SSN trout density models} we used was TU weighting scheme based on watershed area.

shorter geographic distances than did densities along flow-connected pathways from headwaters

Five SSN models were fit to the trout density dataset in R using the SSN package (a copy of the R script is provided as Supplemental B). Three of those models included reach covariates and two models used only an intercept (i.e., mean trout density) with an autocovariance function (Table 2), which was equivalent to ordinary kriging. In all cases, the basic linear mixed model

where $\boldsymbol{y}$ is a vector of measured trout densities, $\mathbf{X}$ is a matrix of covariate values, $\beta$ is a vector of regression coefficients, and $\varepsilon$ is a vector of independent and normally distributed random errors. The spatial structure in residuals was described using vectors of zero-mean random variables $\left(\mathbf{z}_{\mathrm{TU}}, \mathbf{z}_{\mathrm{TD}}\right.$, and $\left.\mathbf{z}_{\mathrm{EUC}}\right)$ with a autocorrelation structure based on tail-up (TU), tail-down (TD), and Euclidean (EUC) covariance functions (Peterson and Ver Hoef 2010, Ver Hoef and Peterson 2010). Each random variable $\left(\mathbf{z}_{\mathrm{TU}}, \mathbf{z}_{\mathrm{TD}}, \mathbf{z}_{\mathrm{EUC}}\right)$ in the autocorrelation structure can be represented by one of several different covariance models (e.g., linear-with-sill, Mariah, exponential, Epanechnikov, spherical models; Chiles and Delfiner 2009, Garreta et al. 2010). Moreover, one or more classes of covariance function (TU, TD, EUC) may be chosen to represent the properties of the stream attribute being modeled (e.g. patterns created by passive downstream diffusion or upstream and downstream movement processes). The choice of covariance function(s) is important because each represents spatial relationships in a different way. The tail-up function restricts correlation to sites that are flow-connected and uses spatial weighting based on userspecified stream attributes (e.g., watershed area, stream order, segment slope) to up- or downweight samples that occur upstream of a location (Frieden et al. 2014). The tail-down function, in contrast, permits correlation between both flow-connected and flow-unconnected locations and a spatial weighting scheme is not necessary. For simplicity, we drew on previous results that suggest a mixed covariance construction usually performs best (Peterson and Ver Hoef 2010; Frieden et al. 2014) and used exponential models for the TD, EUC, and TU functions, with the 
215

216

217

218

219

\section{Block-kriging population estimates}

The SSN models were used to predict trout densities at $100 \mathrm{~m}$ intervals throughout the Salt River network using universal kriging (Cressie 1993). The kriging equations have two parts, predictions based on the linear regression model and adjustments based on local spatial autocorrelation,

$$
\hat{y}\left(s_{0}\right)=\mathbf{x}\left(s_{0}\right)^{\prime} \widehat{\boldsymbol{\beta}}+\mathbf{c}\left(s_{0}\right)^{\prime} \boldsymbol{\Sigma}^{-1}(\boldsymbol{y}-\mathbf{X} \widehat{\boldsymbol{\beta}}),
$$

where $\mathbf{x}\left(s_{0}\right)$ is a vector containing the covariate values at prediction location $s_{0}$ and the vector $\widehat{\boldsymbol{\beta}}$ contains the estimated regression coefficients using REML, so $\mathbf{x}\left(s_{0}\right)^{\prime} \widehat{\boldsymbol{\beta}}$ forms the linear regression prediction. The remaining portion of equation 4 is an adjustment for spatial autocorrelation, where $\mathbf{c}\left(s_{0}\right)$ is a vector of covariances among observed data and the prediction site, and $\boldsymbol{\Sigma}$ is the covariance matrix among observed data. This kriging formulation provides exact interpolations that "honor the data" in contrast to alternatives based on splines (Schabenberger and Gotway 2005). Local prediction variances (Ver Hoef 2008) are given by

$$
\operatorname{vâr}\left[\hat{y}\left(s_{0}\right)\right]=\sigma_{0}^{2}-\mathbf{c}\left(s_{0}\right)^{\prime} \Sigma^{-1} \mathbf{c}\left(s_{0}\right)+\mathbf{d}^{\prime}\left(\mathbf{X}^{\prime} \Sigma^{-1} \mathbf{X}\right)^{-1} \mathbf{d},
$$


240

241

242

243

244

where $\sigma_{0}^{2}=\operatorname{var}\left[y\left(s_{0}\right)\right]$ (including all of the variance components) and $\mathbf{d}=\mathbf{x}\left(s_{0}\right)^{\prime}-$ $\mathbf{X}^{\prime} \boldsymbol{\Sigma}^{-1} \mathbf{c}\left(s_{0}\right)$.

Population estimates were developed from the network predictions using block kriging (BK), which predicts an average value from an integral of a random surface. The mean integral for a portion of a stream network, $B_{0}$, is

$$
\hat{y}\left(B_{0}\right)=\frac{1}{b-a} \int_{a}^{b} y(u) d u
$$

If the integral is over a stream network, then integrals are done piece-wise for each stream segment, added together, and then divided by the total length of integrated stream. In practice, the integral is approximated using a grid of evenly-spaced prediction points along the network. Block-kriging predictions and variances require modification of equations 4 and 5 wherein $\mathbf{c}\left(s_{0}\right)$ is replaced by $\mathbf{c}\left(B_{0}\right)$ and all pairwise covariances are computed between the observed data and the points on the grid used to approximate an integral. Similar modifications are required for $\sigma_{0}^{2}$ in equation 5, and covariates need to be integrated as well. The necessary two-dimensional formulas are given in Ver Hoef (2008), have been adapted for streams (Ver Hoef et al. 2006), and the functionality is included in the SSN package so that BK predictions and variances can be easily generated by users (Ver Hoef et al. 2014).

To approximate the integrals for population estimates in the Salt River network, we created a grid of points at $100 \mathrm{~m}$ intervals throughout the network. The BK estimate of trout density over any network subset then yielded an estimate of the mean trout density, so the population estimate was this density times the length of the network subset. Figure 1 shows the locations where population estimates were made in tributaries and the Salt River mainstem. The same BK procedure was conducted for the full network that supported fish populations to obtain a grand population estimate for the watershed. When making the grand estimate, we excluded downstream sections of some tributaries that are dewatered for irrigation purposes during the summer. As a baseline for comparison, we also derived population estimates for the same areas using a non-spatial SRS estimator with the observed densities (as in classical design-based surveys (Thompson 1992)), which were then expanded based on appropriate stream-length factors.

\section{Results}


269

270

271

272

273

274

275

276

277

278

279

280

281

282

283

284

285

286

287

288

289

290

291

292

293

294

295

296

297

298

Trout densities at the 108 sites ranged from 0 to 132 trout $\cdot 100 \mathrm{~m}^{-1}$ and showed geographic clustering of similar densities (Figure 1) that corroborated the Torgegram results (Figure 2). Densities were usually lowest in the highest elevation stream sites along the eastern portion of the watershed and higher in most western tributaries and the Salt River mainstem. The five SSN models had similar predictive accuracies (LOOCV $\mathrm{r}^{2} \sim 0.49$; RMSPE 21.0) and showed considerable performance gains relative to the MLR $\left(\right.$ LOOCV $\mathrm{r}^{2}=0.19$; $\mathrm{RMSPE}=26.3$; Table 2). Both types of models overestimated low densities and underestimated high densities but the SSN models did so to a lesser degree (Figure 3). The SSN models had AIC scores that were 20-27 points lower than the MLR, despite requiring the estimation of 2-7 additional parameters for the autocovariance functions (Table 2). The temperature covariate was statistically significant in the models where it appeared $(\mathrm{p}<0.05)$ and reach slope was never significant. The canopy covariate was significant in the MLR $(p=0.02)$ but not in the SSNs $(p \geq$ 0.14). Within the SSN model set, SSN3 that used a temperature covariate and TU, TD autocovariance function had the lowest AIC value. Two models without covariates (SSN4 and SSN5) has similar predictive performance as SSN3 but had AIC scores 5-7 points higher. A trout density map predicted using SSN3 showed how abundance varied throughout the network (Figure 4). Noteworthy was that predictions matched observed densities at the 108 sample sites, which is a property of the kriging formulation that was implemented. Also noteworthy was the spatial variation in the size of the prediction standard errors, which were smaller near sample sites because the SSN model used the fitted autocovariance function and local empirical support when making predictions.

Population estimates based on SRS and the five SSN models showed several interesting properties when examined for four representative tributaries (Figure 5). First, SSN-BK estimates could be made for all streams, which was not the case with the SRS estimator in Swift Creek where only one density sample was available (two samples are needed to calculate a variance and confidence interval). Second, results from Willow Creek support the notion that SSN-BK estimates may often be more accurate than those from SRS. Five density samples were available in that stream but only one occurred in the downstream segment where trout densities were high, so the SRS population estimate of $\sim 4,000$ trout was biased low compared to the BK estimate of $\sim 7,000$ trout made from a spatially balanced set of predictions throughout the stream. Third, 
SSN-BK estimates for individual streams were similar regardless of the model chosen, which suggested robustness to different specifications.

A full set of SRS and SSN3-BK population estimates for streams in the Salt River network is provided in Figure 6. The SSN3-BK estimates were usually more precise and showed that eastern tributaries had smaller trout populations $(612-7,128$ trout) than western tributaries (12,963-27,216 trout) and the Salt River mainstem $(42,987+/-11,894$ trout $(95 \% \mathrm{CI}))$. The difference in population estimates was primarily due to the shorter networks that comprised eastern tributaries, but those streams were also especially cold and may have been too unproductive to support high trout densities as indicated by the positive effect of the temperature covariate in the SSN models. The grand SSN3-BK population estimate for the Salt River network was $184,030+/-27,263$ trout; whereas the SRS estimate was $155,828+/-26,514$ trout. Similar to the bias associated with the Willow Creek estimate, the SRS estimate for the full network may have been biased by the large proportion of samples from high elevation tributaries where trout densities were lower (Figure 1). That bias could have been addressed using a stratified random sampling estimator wherein each tributary was treated as a stratum but single samples from some strata would have made variance calculations impossible without ad hoc combinations of multiple streams into workable strata.

\section{Discussion}

Combining fish density surveys and SSN models with broadly available geospatial data frameworks creates a powerful and flexible approach to population estimation for streams and rivers. As we demonstrate, population estimates can be derived at virtually any spatial scale, thereby allowing biological information to be matched with relevant land-uses, landscape features, or jurisdictional and biogeographic boundaries to address conservation and management needs. For example, population estimates at stream or network scales are key for species' conservation assessments (e.g., the 50/500 rule, Franklin 1980), but have rarely been available or are based on extrapolations from a small number of non-random samples (Hilderbrand and Kershner 2000; Cook et al. 2010). Estimates like those developed here for the Salt River basin, which hosted $\sim 150,000$ of the native Yellowstone cutthroat trout (a species of conservation concern), can now be repeated elsewhere to inform status assessments where sufficient data exist. Although 50-100 samples are desirable to estimate parameters for the SSN 
330

331

332

333

334

335

336

337

338

339

340

341

342

343

344

345

346

347

348

349

350

351

352

353

354

355

356

357

358

359

360

models (Isaak et al. 2014), datasets of this size are common within research and management agencies, especially when data are aggregated across multiple projects or agencies. One example, the MARIS database (Multistate Aquatic Resources Information System: www.marisdata.org; Loftus and Beard 2009), contains $>1,000,000$ fisheries surveys for $>1,000$ species in the eastern U.S., an impressive total that nonetheless represents a small fraction of extant data. Another potential application of the SSN-BK estimator was presented by the dewatered stream reaches in the Salt River network, where estimates could have been made for the number of trout those areas would support if perennial flows were restored. Block kriging also has obvious utility for making reference site comparisons used in biological and habitat condition assessments (Kershner and Roper 2010; Hawkins et al. 2010) or within the regulatory arena to determine where standards are exceeded if SSN models are applied to water chemistry attributes (Birkeland 2001).

A key difference between SSN-BK and previous estimators (e.g., Hankin and Reeves 1988; Stevens and Olsen 2004) is that the SSN estimator relies on model-based inference and does not require random sampling (Ver Hoef 2008). Even when designs are randomized, better estimates are often possible using spatial models because random designs have some degree of clustering and ancillary spatial information exists that is useful for estimation (Ver Hoef 2002). The SRS and MLR estimators used in our examples were unweighted, so clustered trout density samples over-represented conditions in some areas and biased results due to spatial unbalance. Although it would have been possible to weight samples in an ad hoc fashion, block-kriging finds an optimal weighting scheme within the blocking area. The SSN-BK estimator is accurate, therefore, because it replaces the average of the observations with an average from an evenlyspaced grid of model predictions that achieves spatial balance. Each prediction is simply a weighted average that has optimality properties, in the sense that it minimizes the mean-squared prediction error (Ver Hoef 2008).

Another important feature of the SSN models is their ability to incorporate covariates and assess effect sizes and statistical significance in the presence of spatial autocorrelation. Although the inclusion of covariates in our Salt River dataset provided only small model improvements, developing fully descriptive density models here was not our goal. Those models are a logical next step, however, and one that will be enhanced by the availability of dozens of reach descriptors for the NHD and NSI networks (Wang et al. 2011; Hill et al. 2016) and the increasing 
361

362

363

364

365

366

367

368

369

370

371

372

373

374

375

376

377

378

379

380

381

382

383

384

385

386

387

388

389

390

391

technical proficiency of users in developing custom covariates (Peterson et al. 2011; Nagel et al. 2014). But as our results also demonstrate, informative covariates are not prerequisite to developing accurate prediction maps with SSN models if datasets are spatially dense and samples are autocorrelated. Those maps can provide detailed information about patterns of abundance and help identify fish density hotspots, which could be useful for directing conservation efforts even without a complete understanding of the processes that create spatial patterns. In the Salt River watershed, for example, the data visualization provided by the prediction maps added considerable depth to our view of the landscape despite a previous familiarity with it. Moreover, the depiction of spatial variation in SSN model prediction precision could be used to guide subsequent sampling efforts to locations that reduced the greatest amount of uncertainty. The Torgegram description of spatial autocorrelation among trout densities might also be useful for designing sampling campaigns in other networks that lack data by providing a first approximation of the stream distances over which samples are partially redundant (Som et al. 2014; Zimmerman and Ver Hoef 2017).

There are three caveats regarding the use of the SSN-BK estimator. First, population estimates for headwater streams will be sensitive to errors associated with the length of stream estimated to support fish, which may be problematic in that headwater reaches are often imprecisely mapped (Bishop et al. 2008). Our familiarity with the Salt River study site allowed us to trim the network based on field observations so that it closely approximated fish habitat, but the size of this reduction was substantial (61\%) and would have inflated population estimates if not addressed. For applications where investigators lack direct knowledge of local conditions, rulesets to trim the network based on defensible criteria should be developed and applied. Two obvious criteria when using the NHDPlus dataset are intermittency and stream slope. In the latter case, fish densities are low in steep reaches (Chisholm and Hubert 1986; Isaak et al. 2017) so exclusion of these areas in mountain landscapes has minor effects on population estimates. In arid landscapes like much of the American West, the network extent shown by NHDPlus is often far more extensive than the actual length of perennial streams, let alone those large enough to support fish populations (Fritz et al. 2013). Intermittent reaches are coded in NHDPlus (McKay et al. 2012), albeit inconsistently in different river basins, so may sometimes be identified and excluded from analysis. A second caveat pertains to preferential sampling and the possibility that strongly clustered sample locations could bias SSN model estimates (Diggle et al. 2010). 
392 393

394

395 396

397

398

399

400

401

402

403

404

405

406

407

408

409

410

411

412

413

414

415

416

417

418

419

420

421

422

Simulation results suggest SSN models perform well with many non-random samples (Falk et al. 2014; Som et al. 2014; Rushworth et al. 2015), but practitioners should always be cautious with ad hoc databases and avoid situations where models are fit to geographically restricted data and then extrapolated across a much larger network extent. In addition to clustered samples, it is desirable to have some sample sites spread throughout the network to encompass a broad range of environmental conditions and ensure that parameter estimates and kriging predictions are robust (Courbois et al. 2008; Elith and Leathwick 2009). The third caveat associated with the SSN-BK estimator is that any systematic bias in local population estimates will translate to broad-scale estimates, and the depletion estimator commonly used in small streams is negatively biased (Cook et al. 2010; Meyer and High 2011). That bias could be remedied by using markrecapture techniques, conducting more electrofishing passes, incorporating detection efficiencies, or applying post-hoc corrections (Peterson et al. 2004; Cook et al. 2010). Accurate local density estimates are desirable but increasing accuracy also comes at a cost when it requires longer sampling durations at individual sites (e.g., mark-recapture estimates). However, if the greatest uncertainty in a broad-scale population estimate stems from sampling a small proportion of the total area, then sampling more sites less accurately could be optimal. That is especially true if the decrease in local accuracy is small, as is often the case with removal estimators because the number of fish captured during the first pass correlates strongly with final estimates based on multiple passes (Cook et al. 2010; Meyer and High 2011). Similar tradeoffs are what ultimately motivated the systematic, broad-scale sampling approach of Hankin and Reeves (1988) and a reexamination of this issue using the spatial statistical simulation capabilities provided in SSN software would be timely (Ver Hoef et al. 2014).

Spatial-stream-network models are powerful tools for stream scientists but the recency of their development also means that work remains to develop additional statistical theory and software that broadens their application. Most relevant to abundance estimation would be SSN models that incorporate habitat-related detection efficiencies (Peterson et al. 2004). However, application of those models, or any others, to large datasets aggregated from many sources face challenges associated with inconsistent field habitat measurement protocols (Millar et al. 2016). Standardization of protocols is needed but geospatial representations of habitat conditions that affect detection efficiency (e.g. stream size, reach slope, habitat complexity) may also be an effective alternative that could be implemented consistently across large areas as stream 
423 covariate databases and remote sensing applications continue to grow (Carbonneau et al. 2012;

424 Hill et al. 2016). Space-time models are another logical extension of SSN models because repeat 425 sampling of sites is fundamental to many fisheries monitoring programs (Thorson et al. 2015).

426 Geostatistical space-time models have been developed for non-network systems (Cressie and

427 Wikle 2011) but their adaption to streams with appropriate covariance structures is a nontrivial 428 task that requires additional research.

429 We are not the first to recognize the potential benefits of geostatistical methods for 430 stream and river data (Ganio et al. 2005; Durance et al. 2006), nor is this the first attempt to use 431 geospatial technologies to derive population estimates at broader scales (Wyatt 2003; Webster et

432 al. 2008). Only recently, however, has the statistical theory for stream networks developed 433 sufficiently (Peterson et al. 2010; Ver Hoef et al. 2010) and been integrated into robust software 434 (Peterson and Ver Hoef 2014; Ver Hoef et al. 2014) to make the methods broadly accessible to 435 users. The timing is opportune given the increasing availability of large, spatially dense fisheries 436 datasets and geospatial frameworks for organizing data (Cooter et al. 2010; McKay et al. 2012). 437 Developing initial SSN-BK population estimates may require a few weeks of work by those with 438 complementary GIS and statistical skills but it then is possible to derive population estimates at 439 any scale within the modeling domain and to later refine population estimates with additional data. The insights yielded by these new spatial analyses regarding the distribution and abundance of stream fishes should prove useful in addressing many conservation and management issues.

\section{Acknowledgements}

444 We thank the Wyoming Game and Fish Department for funding field data collection efforts. M.

445 Hyatt and L. Isaak provided assistance with sampling trout populations in tributary streams. K.

446 Gelwicks and D. Zafft provided trout density data from the Salt River mainstem. D. Isaak was

447 supported by the U.S. Forest Service, Rocky Mountain Research Station during manuscript

448 preparation. Kevin Meyer and three anonymous reviewers provided comments that improved the 449 quality of the final manuscript. 


\section{References}

452 Akaike, H. 1974. A new look at the statistical model identification. IEEE Trans Automat 453 Control. 19(6):716-722. doi: 10.1109/TAC.1974.1100705.

454 Al-Chokhachy, R., Budy, P., and Schaller, H. 2005. Understanding the significance of redd 455 counts: a comparison between two methods for estimating the abundance of and monitoring 456 457 458 bull trout populations. N. Am. J. Fish. Manage. 25(4):1505-1512. doi: 10.1577/M05-006.1.

Beale, C.M., Lennon, J.J., Yearsley, J.M., Brewer, M.J., and Elston, D.A. 2010. Regression analysis of spatial data. Ecol. Lett. 13(2):246-264. doi: 10.1111/j.1461-0248.2009.01422.x.

Birkeland, S. 2001. EPA’s TMDL program. Ecol Law Q 28(2):297-325. doi:

\section{$\underline{10.15779 / Z 38 F 85 M}$.}

Bishop, K., Buffam, I., Erlandsson, M., Fölster, J., Laudon, H., Seibert, J., and Temnerud, J. 2008. Aqua Incognita: the unknown headwaters. Hydrol. Proc. 22(8):1239-1242. doi: 10.1002/hyp.7049.

Brennan, S.R., Torgersen, C.E., Hollenbeck, J.P., Fernandez, D.P., Jensen, C.K., and Schindler, D.E. 2016. Dendritic network models improve isoscapes and quantify influence of landscape and in-stream processes on strontium isotopes in rivers. Geophys. Res. Lett. 43(10):50435051. doi: 10.1002/2016GL068904.

Carbonneau, P., Fonstad, M.A., Marcus, W.A. and Dugdale, S.J. 2012. Making riverscapes real. Geomorphology 137(1):74-86. doi.org/10.1016/j.geomorph.2010.09.030.

Chiles, J.P., and Delfiner, P. 2009. Geostatistics: Modeling Spatial Uncertainty. John Wiley and Sons.

Chisholm, I.M., and Hubert, W.A. 1986. Influence of stream gradient on standing stock of brook trout in the Snowy Range, Wyoming. Northwest Science 60(2):137-139.

Cook, N., Rahel, F.J., and Hubert, W.A. 2010. Persistence of Colorado River Cutthroat Trout populations in isolated headwater streams of Wyoming. Trans. Am. Fish. Soc. 139(5):15001510. doi: 10.1577/T09-133.1.

Cooter, W., Rineer, J., and Bergenroth, B. 2010. A nationally consistent NHDPlus framework for identifying interstate waters: implications for integrated assessments and interjurisdictional TMDLs. Environ. Manage. 46(3):510-524. doi: 10.1007/s00267-010-9526-y.

Courbois, J.Y., Katz, S.L., Isaak, D.J., Steel, E.A., Thurow, R.F., Wargo-Rub, A.M., Olsen, T., Jordan, C.E. 2008. Evaluating probability sampling strategies for estimating redd counts: an 

example with Chinook salmon (Oncorhynchus tshawytscha). Canadian Journal of Fisheries and Aquatic Sciences. 65(9):1814-30. doi: 10.1139/F08-092.

Cressie, N. 1993. Statistics for Spatial Data, Revised Edition. New York: John Wiley and Sons.

Cressie, N., and Wikle, C.K. 2011. Statistics for spatio-temporal data. John Wiley and Sons. Hoboken, New Jersey. 588p.

Diggle, P.J., Menezes, R., Su, T.L. 2010. Geostatistical inference under preferential sampling. Journal of the Royal Statistical Society: Series C (Applied Statistics) 59(2):191-232. doi: 10.1111/j.1467-9876.2009.00701.x.

Dunham, J.B., Rosenberger, A.E., Thurow, R.F., Dolloff, C.A., and Howell, P.J. 2009. Coldwater fish in wadeable streams. Pages 119-138 in Bonar, S.A., Hubert, W.A., Willis, D.W., editors. Standard methods for sampling North American freshwater fishes. American Fisheries Society, Bethesda, Maryland.

Durance, I., Le Pichon, C., and Ormerod, S. J. 2006. Recognizing the importance of scale in the ecology and management of riverine fish. Riv. Res. Appl. 22(10):1143-1152. doi: 10.1002/rra.965.

Elith, J., and Leathwick, J.R., 2009. Species distribution models: ecological explanation and prediction across space and time. Annual Review of Ecology, Evolution, and Systematics 40(1):677-697. doi: 10.1146/annurev.ecolsys.110308.120159.

Falke, J.A., Dunham, J.B., Jordan, C.E., McNyset, K.M., and Reeves, G.H. 2013. Spatial ecological processes and local factors predict the distribution and abundance of spawning by steelhead (Oncorhynchus mykiss) across a complex riverscape. PLoS ONE 8(11): e79232. doi: 10.1371/journal.pone.0079232.

Falk, M.G., McGree, J.M., and Pettitt, A.N. 2014. Sampling designs on stream networks using the pseudo-Bayesian approach. Environ. Ecol. Statist. 21(4):751-773. doi: 10.1007/s10651014-0279-2.

Fausch, K.D., Hawkes, C.L., and Parsons, M.G. 1988. Models that predict standing crop of stream fish from habitat variables: 1950-85. Gen. Tech. Rep. PNW-GTR-213. Portland, OR: U.S. Department of Agriculture, Forest Service, Pacific Northwest Research Station; 52 pages. 
511 Fausch, K.D., Torgersen, C.E., Baxter, C.V., and Li, H.W. 2002. Landscapes to riverscapes:

512 Bridging the gap between research and conservation of stream fishes. BioScience 52(6):483-

513 498. doi: 10.1641/0006-3568(2002)052[0483:LTRBTG]2.0.CO;2.

514 Franklin, I.R. 1980. Evolutionary change in small populations. Pages 135-149 In Soulé, M.E.,

515 and Wilcox, B.A. (Eds.). Conservation Biology: An Evolutionary-Ecological Perspective.

516 Sinauer, Sunderland, MA.

517 Frieden, J.C., Peterson, E.E., Webb, J.A., and Negus, P.M. 2014. Improving the predictive power

518 of spatial statistical models of stream macroinvertebrates using weighted autocovariance

519 functions. Environ. Model. Soft. 60:320-330. doi:10.1016/j.envsoft.2014.06.019.

520 Fritz, K.M. Hagenbuch, E., D’Amico, E., Reif, M., Wigington, P.J. Jr., Leibowitz, S.G.,

521 Comeleo, R.L., Ebersole, J.L., and Nadeau, T. 2013. Comparing the extent and permanence

522

523

524

525

526

527

528

529

530

531

532

533

534

535

536

537

538

539

of headwater streams from two field surveys to values from hydrographic databases and maps. Journal of the American Water Resources Association 49(4): 867-882. doi: 10.1111/jawr.12040.

Ganio, L.M., Torgersen, C.E., and Gresswell, R.E. 2005. A geostatistical approach for describing spatial pattern in stream networks. Front. Ecol. Environ. 3(3):138-144. doi: 10.1890/15409295(2005)003[0138:AGAFDS]2.0.CO;2.

Garreta, V., Monestiez, P. and Ver Hoef, J.M., 2010. Spatial modelling and prediction on river networks: up model, down model or hybrid? Environmetrics 21(5):439-456. doi: 10.1002/env.995.

Gelwicks, K.R., Zafft, D.J., and Gipson, R.G. 2002. Comprehensive study of the Salt River fishery between Afton and Palisades Reservoir from 1995 to 1999 with historical review: fur trade to 1998. Wyoming Game and Fish Department, Fish Division, Cheyenne.

Hankin, D.G., and Reeves, G.H. 1988. Estimating total fish abundance and total habitat area in small streams based on visual estimation methods. Can. J. Fish. Aq. Sci. 45(5):834-844. doi: 10.1139/f88-101.

Hanks, E.M., Schliep, E.M., Hooten, M.B. and Hoeting, J.A. 2015. Restricted spatial regression in practice: geostatistical models, confounding, and robustness under model misspecification. Environmetrics 26(4):243-254. doi: 10.1002/env.2331. 
Hawkins, C.P., Olsen, J.R., and Hill, R.A. 2010. The reference condition: predicting benchmarks for ecological and water-quality assessments. J. N. Am. Benthol. Soc. 29(1):312-343. doi: 10.1899/09-092.1.

Hilborn, R., and Walters, C.J. 1992. Quantitative fisheries stock assessment: choice, dynamics, and uncertainty. Chapman and Hall, New York.

Hilderbrand, R.H., and Kershner, J.L. 2000. Conserving inland cutthroat trout in small streams: how much stream is enough? N. Am. J. Fish. Manage 20(2):513-520. doi: 10.1577/15488675(2000)020<0513:CICTIS>2.3.CO;2.

Hill, R.A., Weber, M.H., Leibowitz, S.G., Olsen, A.R., and Thornbrugh, D.J. 2016. The streamcatchment (StreamCat) dataset: A database of watershed metrics for the conterminous United States. J. Am. Wat. Res. Ass. 52(1):120-128. doi: 10.1111/1752-1688.12372.

Hodges J.S, and Reich B.J. 2010. Adding spatially-correlated errors can mess up the fixed effect you love. The American Statistician 64(4):325-334. doi: 10.1198/tast.2010.10052.

Homer, C.G., Dewitz, J.A., Yang, L., Jin, S., Danielson, P., Xian, G., Coulston, J., Herold, N.D., Wickham, J.D. and Megown, K., 2015. Completion of the 2011 National Land Cover Database for the conterminous United States-Representing a decade of land cover change information. Photogramm. Eng. Remote Sens. 81(5):345-354. doi: 10.14358/PERS.81.5.345.

Isaak, D.J., and Hubert, W.A. 2004. Nonlinear response of trout abundance to summer stream temperatures across a thermally diverse montane landscape. Trans. Am. Fish. Soc. 133(5):1254-1259. doi: 10.1577/T03-010.1.

Isaak, D.J., Luce, C.H., Rieman, B.E., Nagel, D.E., Peterson, E.E., Horan, D.L., Parkes, S., and Chandler, G.L. 2010. Effects of climate change and wildfire on stream temperatures and salmonid thermal habitat in a mountain river network. Ecol. Appl. 20(5):1350-1371. doi: 10.1890/09-0822.1.

Isaak, D.J., Wenger, S.J., Peterson, E.E., Ver Hoef, J.M., Hostetler, S., Luce, C.H., Dunham, J.B., Kershner, J., Roper, B.B., Nagel, D.E., Chandler, G.L., Wollrab, S., Parkes, S., and Horan, D.L. 2016. NorWeST modeled summer stream temperature scenarios for the western U.S. U.S. Forest Service, Rocky Mountain Research Station Research Data Archive. https://doi.org/10.2737/RDS-2016-0033.

Isaak, D.J., Peterson, E.E., Nagel, D.E., Ver Hoef, J.M., and Kershner, J. 2013. A national stream internet to facilitate accurate, high-resolution status and trend assessments for water quality 
571

572

573

574

575

576

577

578

579

580

581

582

583

584

585

586

587

588

589

590

591

592

593

594

595

596

597

598

599

600

parameters and aquatic biotas. U.S. Fish and Wildlife Service, National Landscape

Conservation Cooperative grant. Project website:

www.fs.fed.us/rm/boise/AWAE/projects/NationalStreamInternet.html.

Isaak, D.J., Peterson, E.E., Ver Hoef, J.M., Wenger, S., Falke, J., Torgersen, C., Sowder, C., Steel, A., Fortin, M., Jordan, C., Reusch, A., Som, N., and Monestiez, P. 2014. Applications of spatial statistical network models to stream data. WIRES -Water 1(3):277-294. doi: 10.1002/wat2.1023.

Isaak, D.J., Wenger, S., Young, M.K. 2017. Big biology meets microclimatology: Defining thermal niches of aquatic ectotherms at landscape scales for conservation planning. Ecol. Appl. doi:

Kershner, J.L., and Roper, B.B. 2010. An evaluation of management objectives used to assess stream habitat conditions on federal lands within the interior Columbia Basin. Fisheries 35(6):269-278. doi: 10.1577/1548-8446-35.6.269

Korman, J., Schick, J., and Mossop, B. 2016. Estimating riverwide abundance of juvenile fish populations: How much sampling is enough? N. Am. J. Fish. Manage. 36(2):213-229. doi: 10.1080/02755947.2015.1114542.

Lincoln, F. C. 1930. Calculating waterfowl abundance on the basis of banding returns. United States Department of Agriculture Circular 118:1-4.

Loftus, A.J., and Beard, T.D. 2009. The multistate aquatic resources information system (MARIS): Sharing data across agency boundaries. In: Bonar, S.A.; Hubert, W.A.; Willis, D.W., eds. Standard methods for sampling North American freshwater fishes. Bethesda, MD: American Fisheries Society: 187.

Matheron. G. 1963. Principles of geostatistics. Econ. Geol. 58(8):1246-1266. doi: 10.2113/gsecongeo.58.8.1246.

McKay, L., Bondelid, T., Dewald, T., Johnston, J., Moore, R., and Rea, A. 2012. NHDPlus Version 2: User Guide. ftp://ftp.horizonsystems.com/NHDPlus/NHDPlusV21/Documentation/NHDPlusV2_User_Guide.pdf

Meyer, K.A., and High, B. 2011. Accuracy of removal electrofishing estimates of trout abundance in Rocky Mountain streams. N. Am. J. Fish. Manage. 31(5):923-933. doi: 10.1080/02755947.2011.633684. 
601

602

603

604

605

606

607

608

609

610

611

612

613

614

615

616

617

618

619

620

621

622

623

624

625

626

627

628

629

630

631

Meyer, K.A., Lamansky, J.A., Schill, D.J., and Zaroban, D.W. 2013. Nongame fish species distribution and habitat associations in the Snake River basin of southern Idaho. Western North American Naturalist 73(1):20-34. doi: 10.3398/064.073.0102.

Millar, C.P., Fryer, R.J., Millidine, K.J., Malcolm, I.A. 2016. Modelling capture probability of Atlantic salmon (Salmo salar) from a diverse national electrofishing dataset: Implications for theestimation of abundance. Fisheries Research 177(1):1-12. doi.org/10.1016/j.fishres.2016.01.001.

Moore, R.B., and Dewald, T.G. 2016. The road to NHDPlus-Advancements in digital stream networks and associated catchments. Journal of the American Water Resources Association. doi: $10.1111 / 1752-1688.12389$.

Nagel, D.E., Buffington, J.M., Parkes, S.L., Wenger, S. and Goode, J.R. 2014. A landscape scale valley confinement algorithm: delineating unconfined valley bottoms for geomorphic, aquatic, and riparian applications. Gen. Tech. Rep. RMRS-GTR- 321. Fort Collins, CO: U.S. Department of Agriculture, Forest Service, Rocky Mountain Research Station. 42 p.

Petersen, C.G. 1896. The yearly immigration of young plaice into the Limfjord from the German Sea. Report of the Danish Biological Station 6:5-84.

Peterson, J.T., Thurow, R.F., and Guzevich, J.W., 2004. An evaluation of multipass electrofishing for estimating the abundance of stream-dwelling salmonids. Trans. Am. Fish. Soc. 133(2):462-475. doi: 10.1577/03-044.

Peterson, E.E., and Ver Hoef, J.M. 2010. A mixed-model moving-average approach to geostatistical modeling in stream networks. Ecology 91(3):644-651. doi: 10.1890/08-1668.1.

Peterson E.E, and Ver Hoef, J.M. 2014. STARS: an ArcGIS toolset used to calculate the spatial information needed to fit spatial statistical models to stream network data. J. Stat. Softw. 56(2):1-17. doi: 10.18637/jss.v056.i02.

Peterson, E.E., Sheldon, F., Darnell, R., Bunn, S.E. and Harch, B.D., 2011. A comparison of spatially explicit landscape representation methods and their relationship to stream condition. Freshwater Biology 56(3):590-610. doi: 10.1111/j.1365-2427.2010.02507.x.

Peterson, E.E., Ver Hoef, J.M., Isaak, D.J., Falke, J.A., Fortin, M.J., Jordan, C.E., McNyset, K., Monestiez, P., Ruesch, A.S., Sengupta, A. and Som, N., 2013. Modelling dendritic ecological networks in space: an integrated network perspective. Ecol. Lett. 16(5):707-719. doi: 10.1111/ele.12084. 
632 Pollock, K.H., Nichols, J.D., Brownie, C., and Hines, J.E. 1990. Statistical inference for capture633 recapture experiments. Wildlife Monographs. No. 107.

634 R Development Core Team. 2014. R: A language and environment for statistical computing. R 635 Foundation for Statistical Computing, Vienna, Austria.

636 Rushworth, A.M., Peterson, E.E., Ver Hoef, J.M. and Bowman, A.W. 2015. Validation and 637 638 comparison of geostatistical and spline models for spatial-stream-networks. Environmetrics 26(5):327-338. doi: 10.1002/env.2340.

Schabenberger, O. and Gotway, C.A. 2005. Statistical Methods for Spatial Data Analysis. Chapman and Hall/CRC, Boca Raton, FL.

Schlosser, I.J. 1991. Stream fish ecology: A landscape perspective. BioScience 41(10):704-712. doi: $10.2307 / 1311765$.

Som, N.A., Monestiez, P., Ver Hoef, J.M., Zimmerman, D.L., and Peterson, E.E. 2014. Spatial sampling on streams: Principles for inference on aquatic networks. Environmetrics 25(5):306-323. doi: 10.1002/env.2284.

Stevens, D.L., Jr., and Olsen, A.R. 2004. Spatially balanced sampling of natural resources. J. Am. Stat. Assoc. 99(465):262-278. doi: 10.1198/016214504000000250.

Temesgen, H., and Ver Hoef, J.M. 2015. Evaluation of the spatial linear model, random forest and gradient nearest-neighbor methods for imputing potential productivity and biomass of the Pacific Northwest forests. Forestry 88(1):131-142. doi: 10.1093/forestry/cpu036.

Thompson, S. K. 1992. Sampling. John Wiley \& Sons, New York, NY.

Thorson, J.T., Ianelli, J.N., Munch, S.B., Ono, K., and Spencer, P.D. 2015. Spatial delaydifference models for estimating spatiotemporal variation in juvenile production and population abundance. Can. J. Fish. Aquat. Sci. 72(12): 1897-1915. doi:10.1139/cjfas-20140543.

Torgersen C.E., Baxter C.V., Li, H.W., and McIntosh, B.A. 2006. Landscape influences on longitudinal patterns of river fishes: spatially continuous analysis of fish-habitat relationships. In: Hughes, R.M., Wang, L., and Seelbach, P.W. (eds) Landscape Influences on Stream Habitats and Biological Assemblages, Vol. 48. Bethesda, MD: American Fisheries Society, pp. 473-492. 
661 Turschwell, M.P., Peterson, E.E., Balcombe, S.R., and Sheldon, F. 2016. To aggregate or not?

662 Capturing the spatio-temporal complexity of the thermal regime. Ecol. Indicat. 67:39-48. doi:

663 10.1016/j.ecolind.2016.02.014.

664

Ver Hoef, J.M. 2002. Sampling and geostatistics for spatial data. Ecoscience 9(2):152-161.

665

Ver Hoef, J.M. 2008. Spatial methods for plot-based sampling of wildlife populations. Environ.

666

Ecol. Stat. 15(1):3-13. doi: 10.1007/s10651-007-0035-y.

667

Ver Hoef, J.M., and Peterson, E.E. 2010. A moving average approach for spatial statistical

668

669

models of stream networks. J. Am. Stat. Assoc. 105(489):6-18. doi:

670

10.1198/jasa.2009.ap08248.

Ver Hoef, J.M., Peterson, E.E., and Theobald, D. 2006. Spatial statistical models that use flow

671 and stream distance. Environ. Ecol. Stat. 13(4):449-464. doi: 10.1007/s10651-006-0022-8.

672 Ver Hoef, J.M., Peterson, E.E., Clifford, D., and Shah, R. 2014. SSN: An R package for spatial

673 statistical modeling on stream networks. J. Stat. Softw. 56(3):1-45. doi:

$674 \quad 10.18637 /$ jss.v056.i03.

675 Wang, L., Infante, D., Esselman, P., Cooper, A., Wu, D., Taylor, W., Beard, D., Whelan, G., and 676 Ostroff, A. 2011. A hierarchical spatial framework and database for the national river fish

677

678

679

680

681

682

683

684

685

686

687

688

689 habitat condition assessment. Fisheries 36(9):436-449. doi: 10.1080/03632415.2011.607075.

Webster, R.A., K.H. Pollock, S.K. Ghosh, and Hankin, D.G. 2008. Bayesian spatial modeling of data from unit-count surveys of fish in streams. Trans. Am. Fish. Soc. 137(2):438-453. doi: 10.1577/T06-138.1.

Wenger, S., Isaak, D.J., Luce, C., Neville, H., Fausch, K., Dunham, J., Dauwalter, D., Young, M., Elsner, M., Rieman, B., Hamlet, A., and Williams, J. 2011. Flow regime, temperature, and biotic interactions drive differential declines of Rocky Mountain trout species under climate change. Proc. Nat. Acad. Sci. 108(34):14175-14180. doi: 10.1073/pnas.1103097108.

Wyatt, R.J. 2003. Mapping the abundance of riverine fish populations: integrating hierarchical Bayesian models with a geographic information system (GIS). Can. J. Fish. Aquat. Sci. 60(8): 997-1006. doi: 10.1139/f03-085

Zale, A.V., Parrish, D.L., and Sutton, T.M. 2013. Fisheries techniques, third edition. American Fisheries Society, Bethesda, MD. 1,009 pages. 
690 Zimmerman, D., and Ver Hoef, J. M. 2017. The Torgegram for fluvial variography:

691 characterizing spatial dependence on stream networks. J. Computational Graphical Stat. doi:

$692 \quad 10.1080 / 10618600.2016 .1247006$

693 Zippin, C. 1958. The removal method of population estimation. J. Wild. Manage. 22(1):82-90. 694 doi: $10.2307 / 3797301$. 
695 Table 1. Summary statistics for trout densities and geospatial representations of habitat characteristics at 108 reaches across the Salt

696 River network.

\begin{tabular}{|c|c|c|c|c|c|c|}
\hline Variable & Mean & Median & $\mathrm{SD}$ & Minimum & Maximum & Data source \\
\hline Trout $\cdot 100 \mathrm{~m}^{-1}$ & 32.6 & 25.0 & 29.4 & 0 & 132 & Gelwicks et al. (2002); Isaak and Hubert (2004) \\
\hline $\begin{array}{r}\text { August mean stream } \\
\text { temperature }\left({ }^{\circ} \mathrm{C}\right)\end{array}$ & 11.1 & 11.5 & 2.42 & 5.06 & 15.6 & $\begin{array}{l}\text { NorWeST } \\
\text { (www.fs.fed.us/rm/boise/AWAE/projects/NorWeST.html; }\end{array}$ \\
\hline & & & & & & Isaak et al. 2016) \\
\hline Reach slope $(\%)$ & 3.00 & 2.70 & 2.60 & 0.015 & 10.0 & $\begin{array}{l}\text { NHDPlus Value Added Attribute (www.horizon- } \\
\text { systems.com/NHDPlus/index.php; McKay et al. 2012) }\end{array}$ \\
\hline Canopy $(\%)$ & 30.7 & 31.9 & 16.9 & 0 & 80.1 & $\begin{array}{l}\text { National Land Cover Dataset } \\
\text { (www.mrlc.gov/nlcd2001.php; Homer et al. 2015) }\end{array}$ \\
\hline
\end{tabular}

697 
698 Table 2. Summary statistics for multiple linear regression (MLR) and spatial-stream-network (SSN) models fit to trout density data at 699108 sites in the Salt River network.

\begin{tabular}{|c|c|c|c|c|c|c|c|c|}
\hline Model & Covariates & $b(\mathrm{SE})$ & p-value & Autocovariance & $\mathrm{p}^{*}$ & $\Delta \mathrm{AIC}$ & $\mathrm{CV} \mathrm{r}^{2+}$ & $\mathrm{RMSPE}^{\ddagger}$ \\
\hline \multirow[t]{4}{*}{ MLR } & Intercept & $-55.0(20.5)$ & $<0.01$ & -- & 4 & 27 & 0.19 & 26.3 \\
\hline & Slope & 36.7 (126) & 0.77 & & & & & \\
\hline & Temperature & $6.75(1.43)$ & $<0.01$ & & & & & \\
\hline & Canopy & $0.379(0.163)$ & 0.02 & & & & & \\
\hline \multirow[t]{4}{*}{ SSN1 } & Intercept & $-51.6(29.1)$ & 0.08 & TU, TD & 9 & 1 & 0.49 & 21.0 \\
\hline & Slope & $103(103)$ & 0.32 & & & & & \\
\hline & Temperature & $6.61(2.22)$ & $<0.01$ & & & & & \\
\hline & Canopy & $0.255(0.173)$ & 0.14 & & & & & \\
\hline \multirow[t]{4}{*}{ SSN2 } & Intercept & $-51.4(29.7)$ & 0.09 & TU, TD, EUC & 11 & 5 & 0.49 & 20.9 \\
\hline & Slope & 104 (104) & 0.32 & & & & & \\
\hline & Temperature & $6.60(2.27)$ & $<0.01$ & & & & & \\
\hline & Canopy & $0.249(0.18)$ & 0.16 & & & & & \\
\hline \multirow[t]{2}{*}{ SSN3 } & Intercept & $-18.3(19.1)$ & 0.34 & TU, TD & 7 & 0 & 0.49 & 20.8 \\
\hline & Temperature & 4.57 (1.67) & $<0.01$ & & & & & \\
\hline SSN4 & Intercept & $31.9(5.69)$ & $<0.01$ & TU, TD & 6 & 5 & 0.49 & 20.9 \\
\hline SSN5 & Intercept & $31.4(9.00)$ & $<0.01$ & TU, TD, EUC & 8 & 7 & 0.50 & 20.5 \\
\hline
\end{tabular}

700 Number of model parameters. In addition to covariate parameters, SSN models include 3-7 parameters associated with the 701 autocovariance construction (Ver Hoef and Peterson 2010).

$702{ }^{\dagger}$ Squared correlation between the leave-one-out cross-validation prediction and observed trout densities.

$703 \quad{ }^{\ddagger}$ Root mean square prediction error. 
Fig. 1. Salt River watershed in the western U.S. and locations of trout density estimates at 108 sites. Population estimates were subsequently made for areas upstream of the green bars on tributaries and downstream of the green bar on the Salt River mainstem.

Fig. 2. Empirical Torgegram describing patterns in spatial similarity among trout densities at 108 sites. Symbol sizes are proportional to the number of data pairs averaged for each semivariance value.

Fig. 3. Comparison of leave-one-out cross validation (LOOCV) predictions for trout density derived from a multiple linear regression (A) and a spatial-stream-network model (SSN3, B). Dashed line indicates 1:1 relationship.

Fig. 4. Trout density map predicted by universal kriging and a spatial-stream-network model (SSN3) fit to 108 samples. Stream lines are colored by predicted values and the width of the black stream border is proportional to prediction standard errors. Population estimates were made for areas upstream of the green bars on tributaries and downstream of the green bar on the Salt River mainstem. Predictions were not made in the downstream extents of several eastern tributaries and an upper section of the Salt River where channels are dewatered during the summer.

Fig. 5. Trout population estimates for four tributary streams derived from simple random sample (SRS) and spatial-stream-network (SSN) block-kriging estimators. Error bars denote 95\% confidence intervals; sample sizes are the number of fish density surveys conducted within each tributary. A SRS estimate was not possible for Swift Creek where a single site was sampled.

Fig. 6. Trout population estimates from simple random sample (SRS) and spatial-stream-network (SSN3) block-kriging estimators for the Salt River mainstem and tributary streams draining the western (A) and eastern (B) sides of the watershed. Error bars denote 95\% confidence intervals; SRS estimates were not possible for Strawberry Creek and Swift Creek where single sites were sampled. 


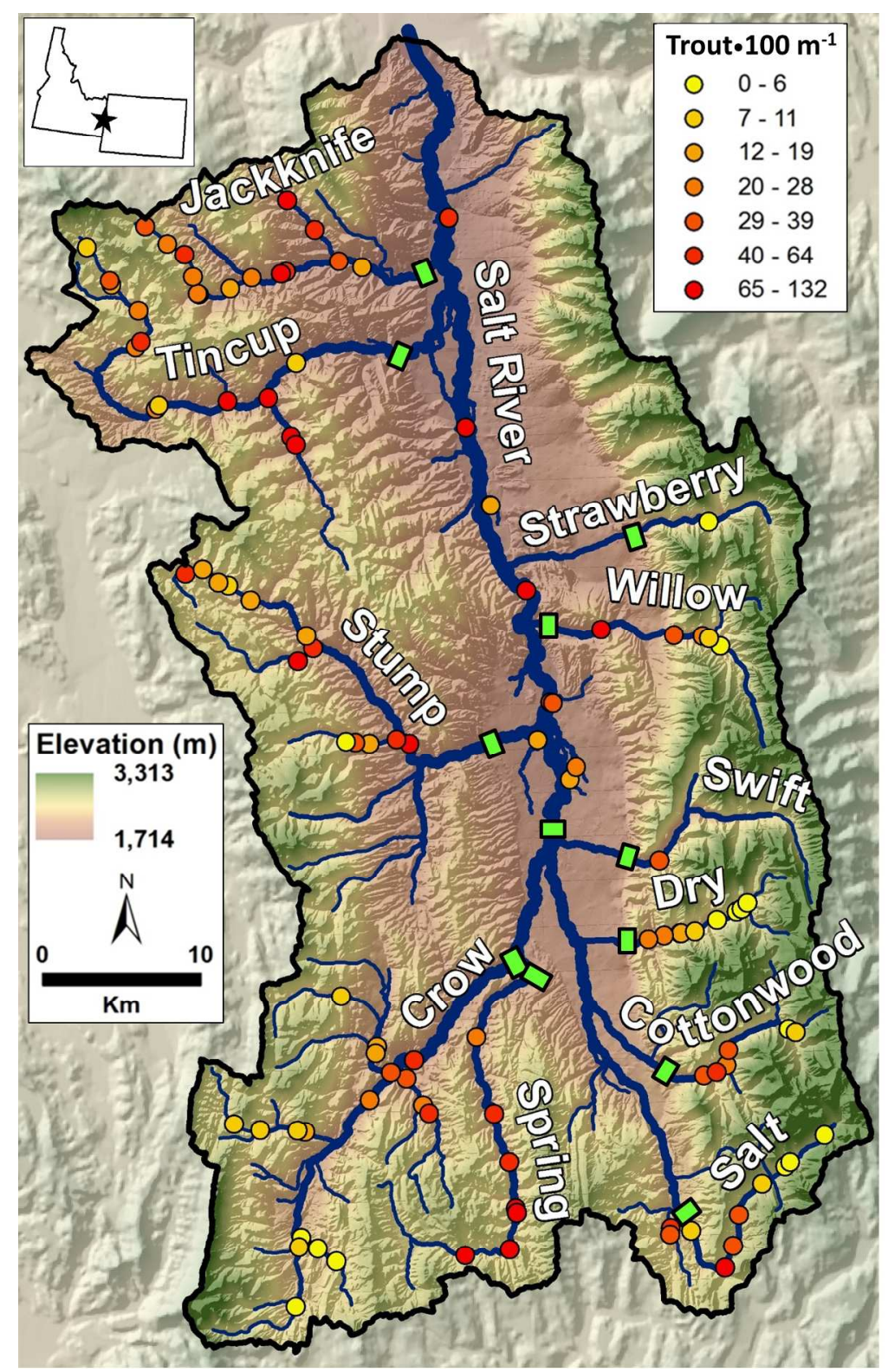

Figure 1.

fig 1

$190 \times 254 \mathrm{~mm}(300 \times 300$ DPI $)$ 


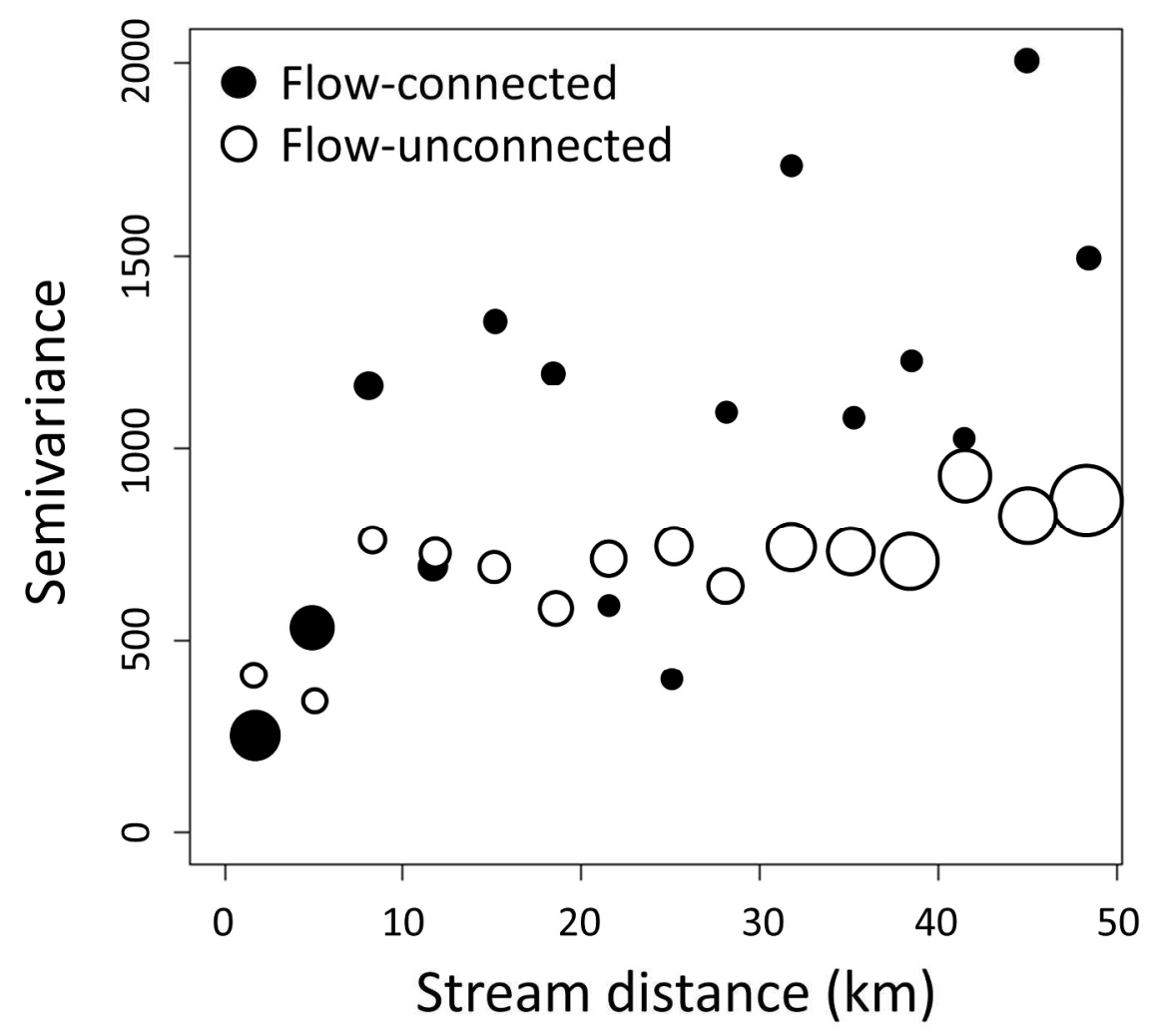

Figure 2.

Fig 2

$190 \times 254 \mathrm{~mm}(300 \times 300 \mathrm{DPI})$ 


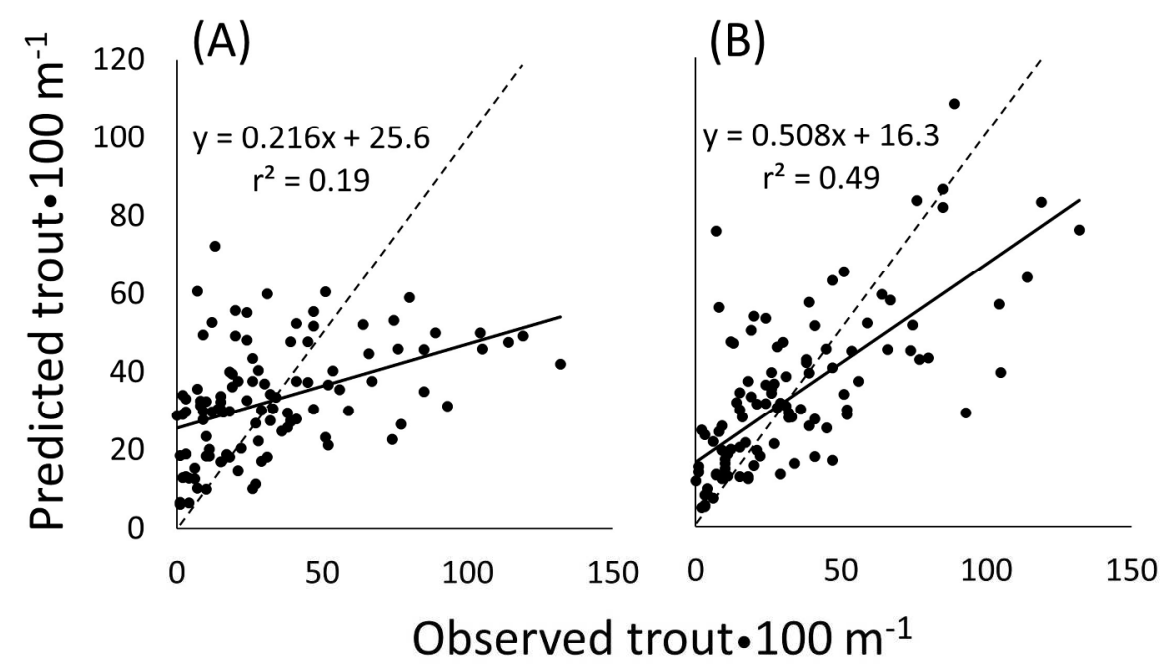

Figure 3.

Fig 3

$190 \times 254 \mathrm{~mm}(300 \times 300$ DPI) 


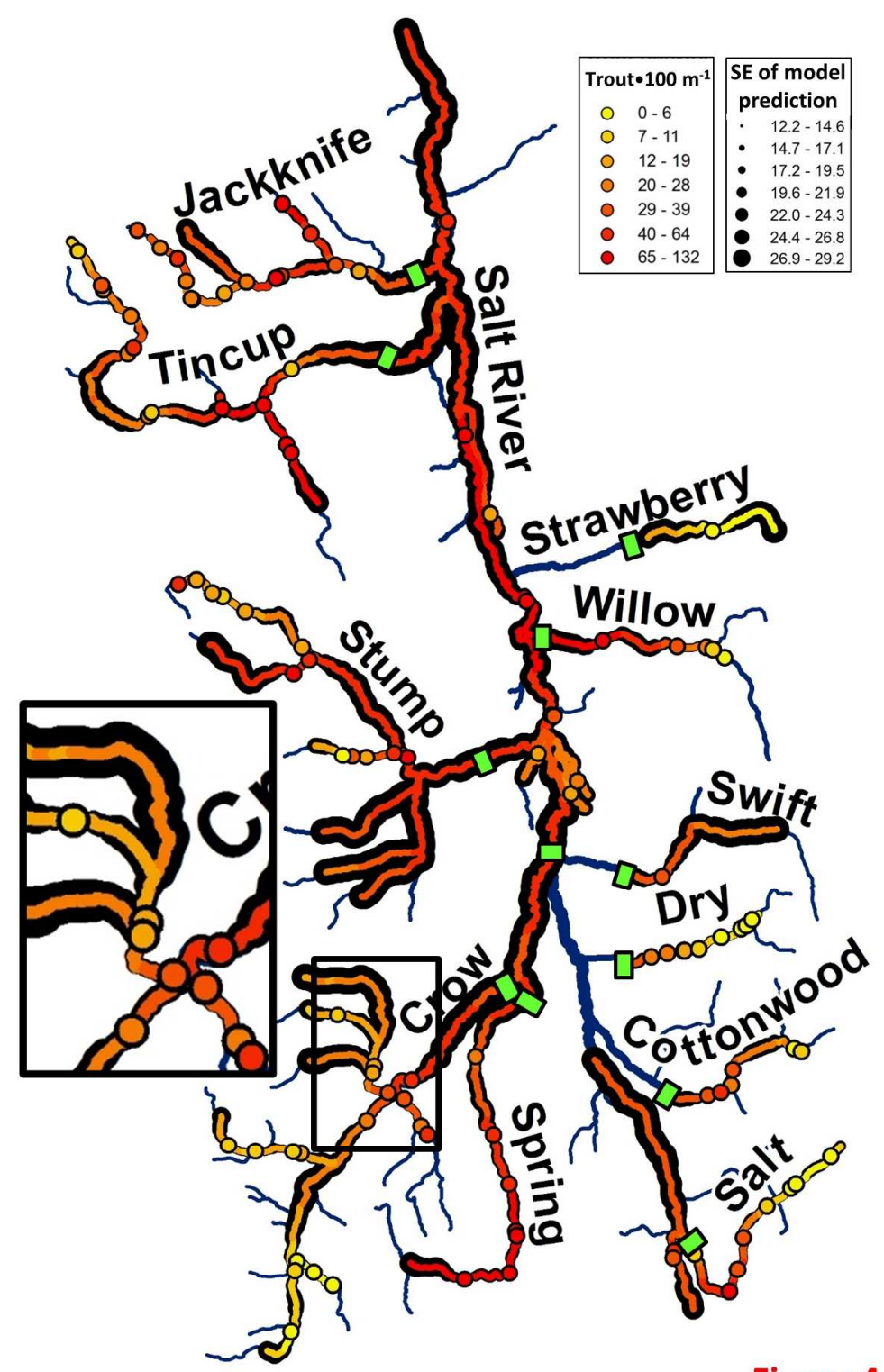

Figure 4.

fig 4

$190 \times 254 \mathrm{~mm}(300 \times 300$ DPI $)$ 


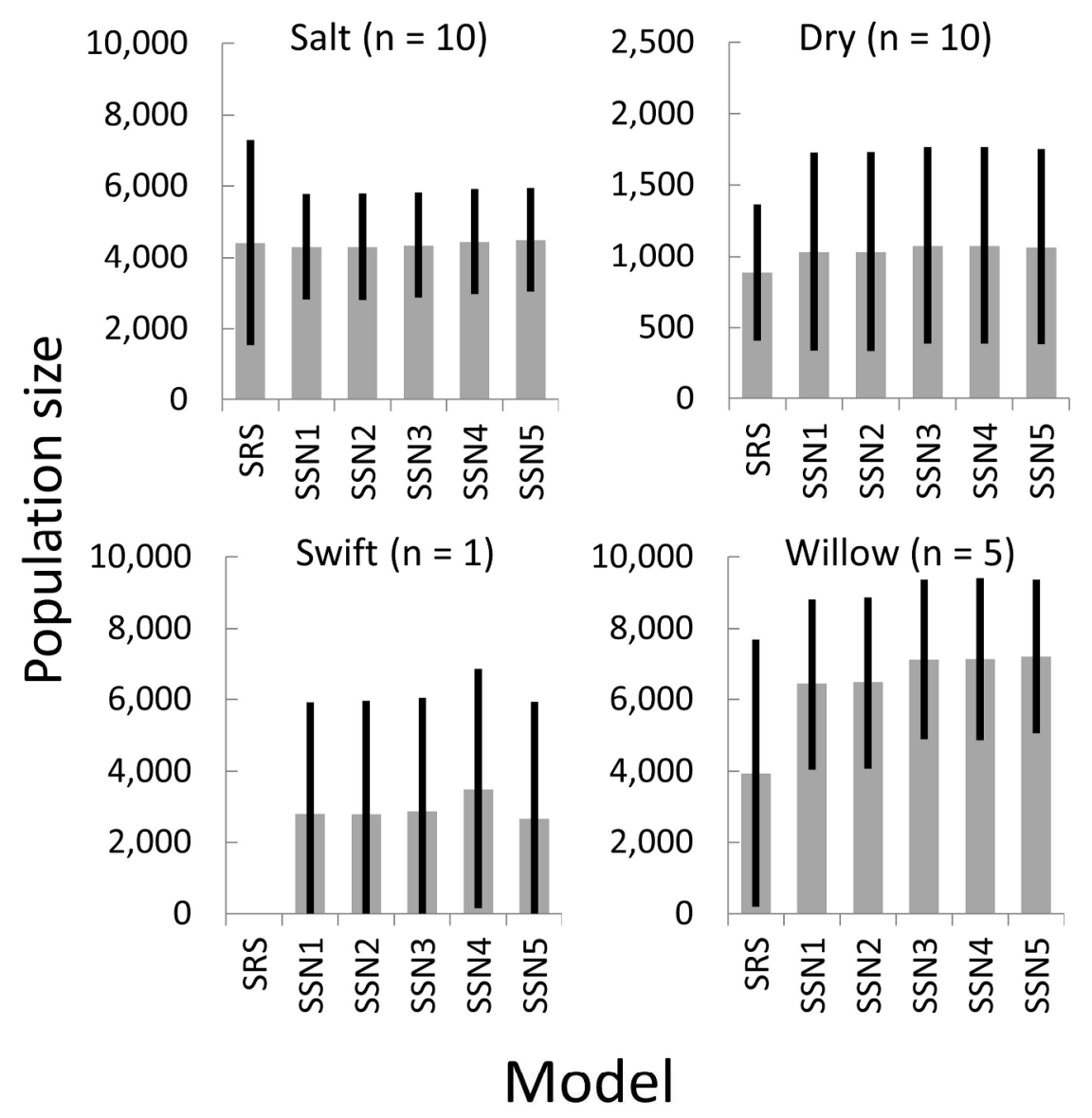

Figure 5.

fig 5

$190 \times 254 \mathrm{~mm}(300 \times 300 \mathrm{DPI})$ 


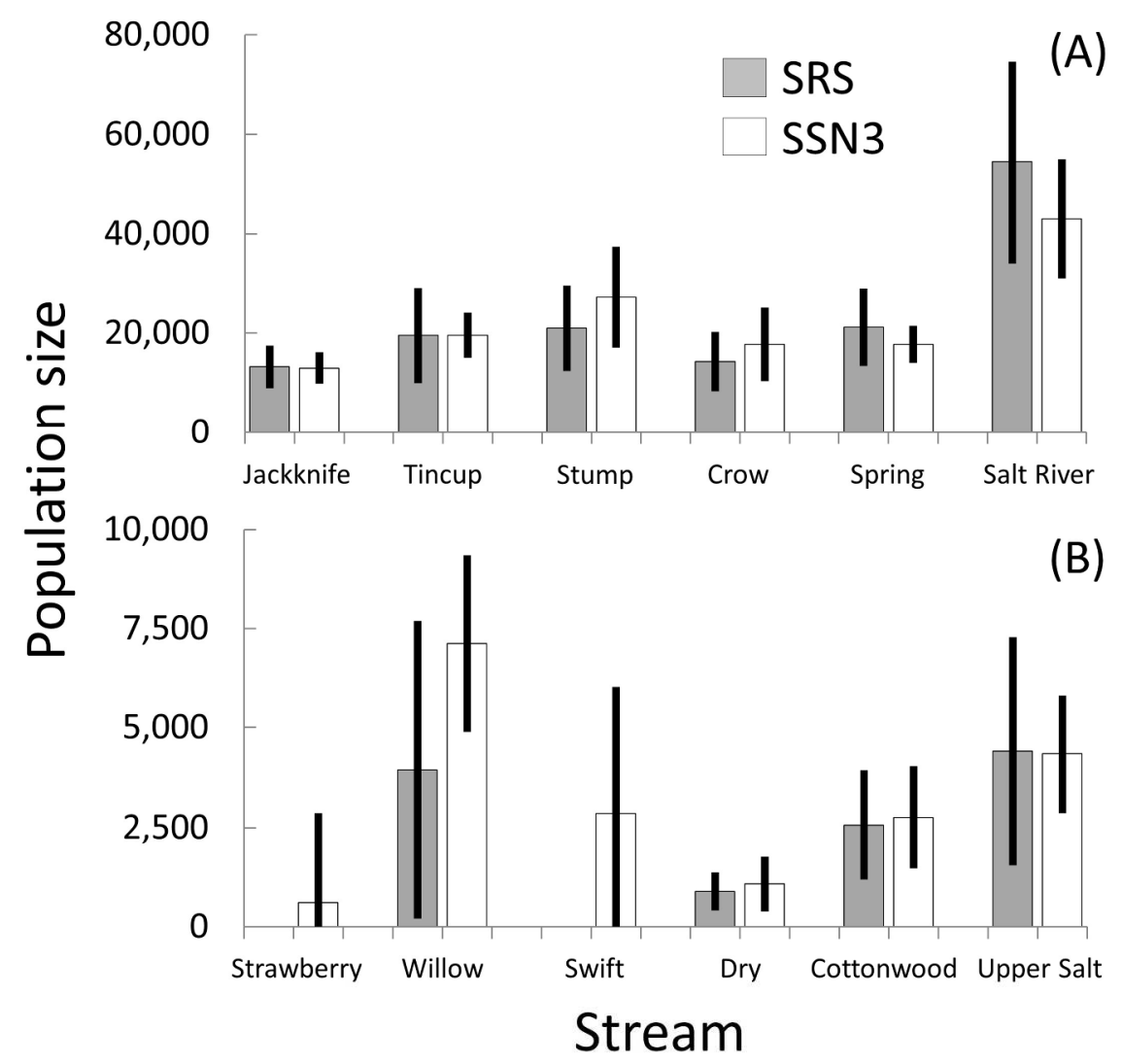

Figure 6.

fig 6

$190 \times 254 \mathrm{~mm}(300 \times 300 \mathrm{DPI})$ 
Supplemental A. ZIP file containing the LSN object file "LSN_TroutDensity_BlockKrige.ssn" and ESRI geodatabase "LSN_TroutDensity_BlockKrige.mdb" to replicate the Salt River analysis is downloadable at the SSN/STARS website "Software and Data" subpage (www.fs.fed.us/rm/boise/AWAE/projects/SSN_STARS/software_data.html).

Supplemental B. Annotated R script "SaltRiver_TroutDensity_BlockKriging.R” used to model trout densities in the Salt River and derive block-kriging population estimates with the LSN object file from Appendix 1.

\#Load SSN package into $R$ library("SSN")

\#Set working directory to location of .ssn directory setwd("C:II...")

\#import the data from the .ssn directory and create a SpatialStreamNetwork object with basic set of prediction points for all reach midpoints

SaltWQ <- importSSN("lsndata/LSN_TroutDensity_BlockKrige.ssn", predpts = "preds")

\#Import prediction points spaced at 100m intervals for block-kriging estimates of individual streams

SaltWQ <- importPredpts(SaltWQ, "Cottonwood", "ssn")

SaltWQ <- importPredpts(SaltWQ, "Crow", "ssn")

SaltWQ <- importPredpts(SaltWQ, "Dry", "ssn")

SaltWQ <- importPredpts(SaltWQ, "Jackknife", "ssn")

SaltWQ <- importPredpts(SaltWQ, "Salt", "ssn")

SaltWQ <- importPredpts(SaltWQ, "Spring", "ssn")

SaltWQ <- importPredpts(SaltWQ, "Strawberry", "ssn")

SaltWQ <- importPredpts(SaltWQ, "Stump", "ssn")

SaltWQ <- importPredpts(SaltWQ, "Swift", "ssn")

SaltWQ <- importPredpts(SaltWQ, "Tincup", "ssn")

SaltWQ <- importPredpts(SaltWQ, "Willow", "ssn")

SaltWQ <- importPredpts(SaltWQ, "SaltRiver", "ssn")

\#Import prediction points spaced at 100 m intervals for block-kriging estimate of full network SaltWQ <- importPredpts(SaltWQ, "Network", "ssn")

\#Create distance matrices among stream prediction points createDistMat(SaltWQ, predpts = "preds", o.write = TRUE, amongpreds $=$ TRUE) createDistMat(SaltWQ, predpts = "Cottonwood", o.write $=$ TRUE, amongpreds $=$ TRUE) createDistMat (SaltWQ, predpts = "Crow", o.write $=$ TRUE, amongpreds $=$ TRUE) createDistMat (SaltWQ, predpts = "Dry", o.write $=$ TRUE, amongpreds $=$ TRUE) 
createDistMat(SaltWQ, predpts = "Jackknife", o.write $=$ TRUE, amongpreds $=$ TRUE) createDistMat (SaltWQ, predpts = "Salt", o.write $=$ TRUE, amongpreds $=$ TRUE) createDistMat(SaltWQ, predpts $=$ "Spring", o.write $=$ TRUE, amongpreds $=$ TRUE) createDistMat(SaltWQ, predpts = "Strawberry", o.write = TRUE, amongpreds $=$ TRUE) createDistMat (SaltWQ, predpts = "Stump", o.write = TRUE, amongpreds $=$ TRUE) createDistMat(SaltWQ, predpts = "Swift", o.write = TRUE, amongpreds $=$ TRUE) createDistMat(SaltWQ, predpts = "Tincup", o.write $=$ TRUE, amongpreds $=$ TRUE) createDistMat(SaltWQ, predpts = "Willow", o.write = TRUE, amongpreds $=$ TRUE) createDistMat(SaltWQ, predpts = "SaltRiver", o.write $=$ TRUE, amongpreds $=$ TRUE)

\#Create distance matrix among network prediction points (calculations require a few minutes) createDistMat (SaltWQ, predpts = "Network", o.write = TRUE, amongpreds = TRUE)

\#Describe the names of the variables in the point.data data.frame for each observed and prediction data set names $($ SaltWQ)

\#plot Salt River network and locations of 108 trout density observations plot(SaltWQ, lwdLineCol = "afvArea", lwdLineEx = 5, lineCol = "blue", pch $=19, x l a b=$ "x-coordinate $(m) ", y l a b=" y$-coordinate $(m) "$, asp $=1)$

\#plot values of 108 trout density observations (Figure 1) brks <-plot(SaltWQ, "trout_100m", lwdLineCol= "afvArea", lwdLineEx $=5$, lineCol = "black", xlab = "x-coordinate", $y l a b=" y$-coordinate", asp=1)

\#plot Torgegram based on 108 trout density observations (Figure 2) SaltWQ.Torg <- Torgegram (SaltWQ, "trout_100m", nlag $=15$, nlagcutoff $=1$, maxlag $=50000)$ plot(SaltWQ.Torg)

\#Fit nonspatial multiple linear regression (MLR) in Table 2 SaltWQ.glmssn0 <-glmssn(trout_100m SLOPE + S1_93_11+CANOPY, SaltWQ, CorModels $=$ NULL, use.nugget $=$ TRUE, EstMeth $=" R E M L ")$ summary(SaltWQ.glmssn0)

\#Fit SSN1 in Table 2.

SaltWQ.glmssn 1 <-glmssn(trout_100m SLOPE + S1_93_11 + CANOPY, SaltWQ, CorModels $=c($ "Exponential.tailup", "Exponential.taildown"), addfunccol = "afvArea", EstMeth = "REML")

summary(SaltWQ.glmssn1)

\#Fit SSN2 in Table 2.

SaltWQ.glmssn $2<-$ glmssn(trout_100m SLOPE + S1_93_11+CANOPY, SaltWQ, CorModels $=c($ "Exponential.tailup", "Exponential.taildown", "Exponential.Euclid"), addfunccol = "afvArea", EstMeth = "REML") summary(SaltWQ.glmssn2) 
\#Fit SSN3 in Table 2.

SaltWQ.glmssn3 <-glmssn(trout_100m $\sim$ S1_93_11, SaltWQ,

CorModels $=c($ "Exponential.tailup", "Exponential.taildown"),

addfunccol = "afvArea", EstMeth = "REML")

summary(SaltWQ.glmssn3)

\#Fit SSN4 in Table 2.

SaltWQ.glmssn4 <- glmssn(trout_100m 1, SaltWQ,

CorModels $=c($ "Exponential.tailup", "Exponential.taildown"),

addfunccol = "afvArea", EstMeth = "REML")

summary(SaltWQ.glmssn4)

\#Fit SSN5 in Table 2.

SaltWQ.glmssn5 <- glmssn(trout_100m 1, SaltWQ,

CorModels $=c($ "Exponential.tailup", "Exponential.taildown", "Exponential.Euclid"),

addfunccol = "afvArea", EstMeth = "REML")

summary(SaltWQ.glmssn5)

\#Report AIC values (Use ML instead of REML in above model fits to estimate correct AIC values)

AIC(SaltWQ.glmssn0)

AIC(SaltWQ.glmssn1)

AIC(SaltWQ.glmssn2)

AIC(SaltWQ.glmssn3)

AIC(SaltWQ.glmssn4)

AIC(SaltWQ.glmssn5)

\#Report cross-validation statistics and confidence intervals

CrossValidationStatsSSN(SaltWQ.glmssn0)

CrossValidationStatsSSN(SaltWQ.glmssn1)

CrossValidationStatsSSN(SaltWQ.glmssn2)

CrossValidationStatsSSN(SaltWQ.glmssn3)

CrossValidationStatsSSN(SaltWQ.glmssn4)

CrossValidationStatsSSN(SaltWQ.glmssn5)

\#Report variance composition among covariate effects and autocovariance functions varcomp (SaltWQ.glmssn0)

varcomp (SaltWQ.glmssn 1)

varcomp (SaltWQ.glmssn2)

varcomp (SaltWQ.glmssn3)

varcomp (SaltWQ.glmssn4)

varcomp(SaltWQ.glmssn5)

\#Plot graphs of leave-one-out cross-validation (LOOCV) predictions \& SES cv.out <- CrossValidationSSN(SaltWQ.glmssn2) 
$\operatorname{par}(m$ row $=c(1,1))$

plot(SaltWQ.glmssn2\$sampinfo\$z,

cv.out[, "cv.pred"], pch = 19,

$x l a b=$ "Observed Data", ylab = "LOOCV Prediction")

\#Save LOOCV predictions \& SES to working directory file

write.csv(cv.out, "cv_out_trout100m.csv", row.names $=$ FALSE)

\#Calculate \& plot model residuals \& influence measures

resids $<$-residuals(SaltWQ.glmssn2)

class(resids)

resids.df<- getSSNdata.frame(resids)

names(resids.df)

plot(resids)

hist(resids, xlab = "Raw Residuals")

qqnorm(resids)

\#Save residuals \& influence measures to working directory file

write.csv(resids.df, "resids_trout100m.csv", row.names = FALSE)

\#Plot 108 observation sites as large circles prior to block-kriging prediction points plot(SaltWQ, "trout_100m", pch =1, cex $=3$,

$x l a b=$ "x-coordinate $(m) ", y$ lab $=$ "y-coordinate $(m) "$,

$x \lim =c(1715000,1770000), y \lim =c(1370000,1450000))$

\#Plot 100 m prediction points for individual stream blocks

SaltWQ.glmssn2.cottonwood <- predict(SaltWQ.glmssn2, "Cottonwood")

plot(SaltWQ.glmssn2.cottonwood, "trout_100m", add = TRUE,

$x \lim =c(1715000,1770000), y \lim =c(1370000,1450000))$

SaltWQ.glmssn2.crow <- predict(SaltWQ.glmssn2, "Crow")

plot(SaltWQ.glmssn2.crow, "trout_100m", add = TRUE,

$x \lim =c(1715000,1770000), y \lim =c(1370000,1450000))$

SaltWQ.glmssn2.dry <- predict(SaltWQ.glmssn2, "Dry")

plot(SaltWQ.glmssn2.dry, "trout_100m", add = TRUE,

$x \lim =c(1715000,1770000), y \overline{l i m}=c(1370000,1450000))$

SaltWQ.glmssn2.jackknife <- predict(SaltWQ.glmssn2, "Jackknife")

plot(SaltWQ.glmssn2.jackknife, "trout_100m", add = TRUE,

$x \lim =c(1715000,1770000), y \lim =\bar{c}(1370000,1450000))$

SaltWQ.glmssn2.salt <-predict(SaltWQ.glmssn2, "Salt")

plot(SaltWQ.glmssn2.salt, "trout_100m", add = TRUE,

xlim $=c(1715000,1770000), y \overline{l i m}=c(1370000,1450000))$

SaltWQ.glmssn2.spring <- predict(SaltWQ.glmssn2, "Spring")

plot(SaltWQ.glmssn2.spring, "trout_100m", add = TRUE,

$x \lim =c(1715000,1770000), y \lim =c(1370000,1450000))$

SaltWQ.glmssn2.strawberry <- predict(SaltWQ.glmssn2, "Strawberry")

plot(SaltWQ.glmssn2.strawberry, "trout_100m", add = TRUE, 


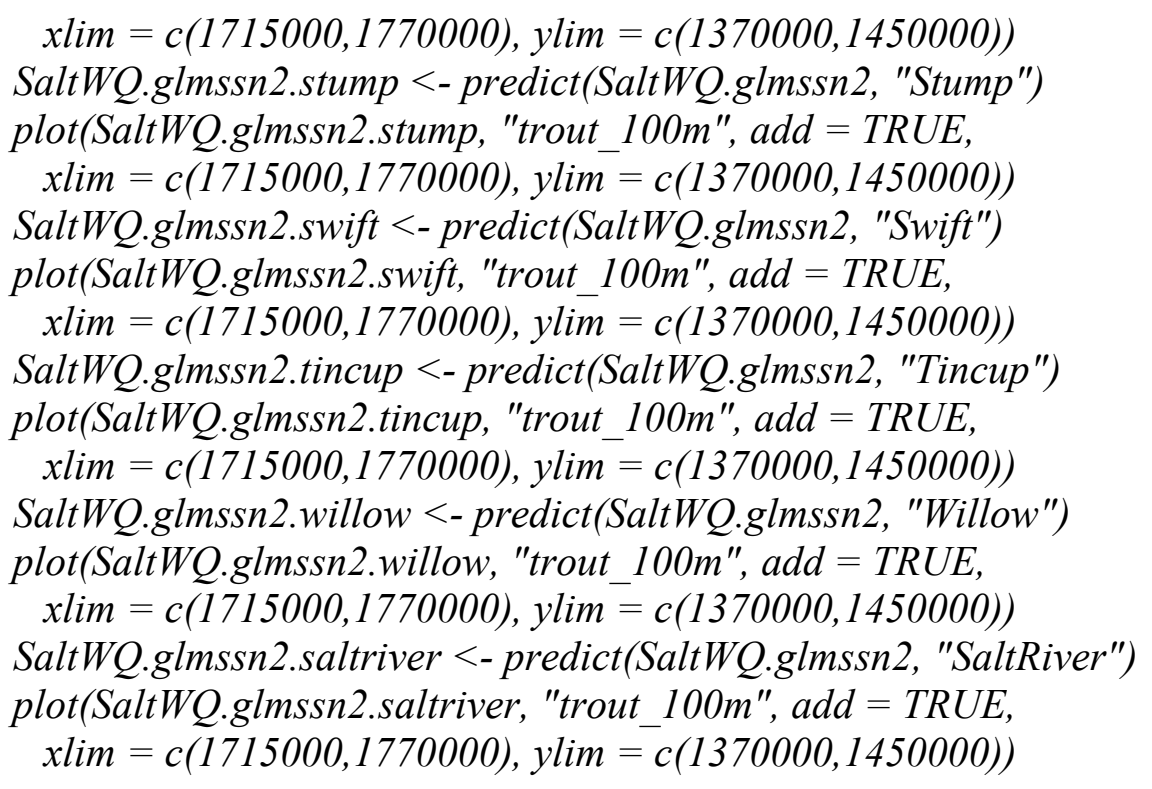

\#Obtain block-kriging estimates of mean trout density \& SEs for individual stream blocks SaltWQ.glmssn2.cottonwood <- BlockPredict(SaltWQ.glmssn2, "Cottonwood")

SaltWQ.glmssn2.cottonwood

SaltWQ.glmssn2.crow <- BlockPredict(SaltWQ.glmssn2, "Crow")

SaltWQ.glmssn2.crow

SaltWQ.glmssn2.dry <- BlockPredict(SaltWQ.glmssn2, "Dry")

SaltWQ.glmssn2.dry

SaltWQ.glmssn2.jackknife <- BlockPredict(SaltWQ.glmssn2, "Jackknife")

SaltWQ.glmssn2.jackknife

SaltWQ.glmssn2.salt <- BlockPredict(SaltWQ.glmssn2, "Salt")

SaltWQ.glmssn2.salt

SaltWQ.glmssn2.spring <- BlockPredict(SaltWQ.glmssn2, "Spring")

SaltWQ.glmssn2.spring

SaltWQ.glmssn2.strawberry <- BlockPredict(SaltWQ.glmssn2, "Strawberry")

SaltWQ.glmssn2.strawberry

SaltWQ.glmssn2.stump <- BlockPredict(SaltWQ.glmssn2, "Stump")

SaltWQ.glmssn2.stump

SaltWQ.glmssn2.swift <- BlockPredict(SaltWQ.glmssn2, "Swift")

SaltWQ.glmssn2.swift

SaltWQ.glmssn2.tincup <- BlockPredict(SaltWQ.glmssn2, "Tincup")

SaltWQ.glmssn2.tincup

SaltWQ.glmssn2.willow <- BlockPredict(SaltWQ.glmssn2, "Willow")

SaltWQ.glmssn2.willow

SaltWQ.glmssn2.SaltRiver <- BlockPredict(SaltWQ.glmssn2, "SaltRiver")

SaltWQ.glmssn2.SaltRiver

\#Save values of block predictions \& SEs at 100 m prediction points to working directory file SaltWQ.cottonwood <- predict(SaltWQ.glmssn2, "Cottonwood")

predldf<- getSSNdata.frame(SaltWQ.cottonwood, "Cottonwood") 
write.csv(pred1df, "SaltWQ_trout100m_SSN2_cottonwood_BlockPredictions.csv", row.names = FALSE)

\#Plot 100 m prediction points for full network

SaltWQ.glmssn2.network<-predict(SaltWQ.glmssn2, "Network")

plot(SaltWQ.glmssn2.network, "trout_100m", add = TRUE,

$x \lim =c(1715000,1770000), y \lim =c(1370000,1450000))$

\#Obtain block-kriging estimates of mean trout density \& SEs for full network (calculation requires several minutes)

SaltWQ.glmssn2.network <- BlockPredict(SaltWQ.glmssn2, "Network")

SaltWQ.glmssn2.network

\#Plot predictions for full network at reach midpoints with symbol size inverse to SES

SaltWQ.preds <- predict(SaltWQ.glmssn2, "preds")

plot(SaltWQ.preds, SEcex.max $=1.4$, SEcex.min $=.7 / 3 * 2$,

breaktype = "user", brks = brks)

\#Is this cool or what? 